Revisión

\title{
Historia y perspectivas del modelo GGAVATT (Grupos Ganaderos de Validación y Transferencia de Tecnología)
}

Heriberto Román Ponce ${ }^{\mathrm{a}}$

Miguel Arcangel Rodríguez Chessani ${ }^{\text {b }}$

José Antonio Espinosa García ${ }^{\mathrm{c}}$

Tomás Arturo González Orozco ${ }^{\mathrm{d}}$

Alejandra Vélez Izquierdo ${ }^{c}$

Juan Prisciliano Zárate Martínez ${ }^{\mathrm{a} *}$

Martha Eugenia Valdovinos Terán ${ }^{a}$

Rubén Cristino Aguilera Sosa ${ }^{\text {a }}$

Rafael Guarneros Altamirano ${ }^{\text {e }}$

Rubén Santos Echeverría ${ }^{\mathrm{f}}$

Héctor Macario Bueno Díaz ${ }^{\text {b }}$

Ubaldo Aguilar Barradas $^{b}$

${ }^{a}$ Instituto Nacional de Investigaciones Forestales Agrícolas y Pecuarias. CIR Golfo Centro, Campo Experimental la Posta. Km 22.5, carretera federal Veracruz-Córdoba, 94277, Paso del Toro, Municipio de Medellín, Veracruz, México.

${ }^{\mathrm{b}}$ Universidad Veracruzana. Facultad de Medicina Veterinaria y Zootecnia, Veracruz, Ver., México.

${ }^{\mathrm{c}}$ Instituto Nacional de Investigaciones Forestales Agrícolas y Pecuarias. CENID Fisiología, Ajuchitlán, Querétaro, México.

${ }^{\mathrm{d}}$ Instituto Nacional de Investigaciones Forestales Agrícolas y Pecuarias. CIR Centro, Campo Experimental Bajío, Celaya, Guanajuato, México. 
${ }^{\mathrm{e}}$ Instituto Nacional de Investigaciones Forestales Agrícolas y Pecuarias. CIR Noreste, Sitio Experimental Aldama, Aldama, Tamaulipas, México.

${ }^{\mathrm{f}}$ Instituto Nacional de Investigaciones Forestales Agrícolas y Pecuarias. CIR Pacífico Sur, Campo Experimental Iguala, Iguala, Guerrero, México.

*Autor de correspondencia: zarate.juan@inifap.gob.mx

\section{Resumen:}

El objetivo del presente documento es revisar las experiencias obtenidas con el GGAVATT desde su formación. Para lo cual se llevó a cabo una revisión de fuentes secundarias que reportan resultados, experiencias e impactos del desarrollo e implementación del modelo. Se identificaron cinco etapas: de 1970-1982, poniendo los cimientos; de 1983-1989 formación del modelo; de 1990-1996 validación del modelo; de 1997-2007 expansión nacional; de 2008 a la fecha adaptación y sobrevivencia. Se presentan los resultados obtenidos en cada una de estas etapas y la participación en diferentes proyectos asociados a los programas oficiales del sector en diferentes estados del país. En todos los proyectos en donde la metodología GGAVATT se ha aplicado adecuadamente, los resultados técnicos, económicos, sociales y ecológicos, han sido positivos. Se conoce ahora cuales son los factores de éxito que proporcionan buenos resultados y se dispone de una estrategia que puede ser de gran utilidad para mejorar la situación de los pequeños y medianos productores pecuarios de México.

Palabras clave: Modelo de transferencia, Innovación, Ganadería, Adopción de tecnología, Impactos.

Recibido: 10/11/2020

Aceptado: 06/01/2021

\section{Introducción}

El Instituto Nacional de Investigaciones Forestales Agrícolas y Pecuarias (INIFAP) y las instituciones de enseñanza superior han generado tecnología con potencial para duplicar o triplicar la producción de carne, leche y miel en las principales regiones agroecológicas del país. Adicionalmente, el INIFAP desarrolló e implementó en las últimas cuatro décadas un modelo de transferencia de tecnología llamado Grupos Ganaderos de Validación y 
Transferencia de Tecnología (GGAVATT), el cual consiste en una estrategia que facilita el proceso de transferencia de tecnología, con el propósito de incrementar la productividad y rentabilidad de las unidades de producción, para contribuir a mejorar el nivel y calidad de vida de los productores y como consecuencia de la sociedad en general ${ }^{(1)}$.

Los objetivos específicos del modelo son: inducir la organización de los ganaderos en grupos con objetivos comunes; lograr cambios de actitud, comportamiento y aptitud en los productores que los induzcan al uso y adopción de tecnología; aumentar la productividad de las Unidades de Producción Pecuaria (UPP), para que sean rentables, competitivas y sustentables; mejorar el nivel de vida de los productores y sus familias; fomentar la conservación y el aprovechamiento óptimo de los recursos naturales; fortalecer la integración de las cadenas de valor pecuario; y retroalimentar con demandas y problemas tecnológicos a las instituciones de investigación y docencia ${ }^{(1)}$.

Este modelo ha demostrado ser aplicable para facilitar la transferencia de tecnología pecuaria; por ello el objetivo del presente documento es el de revisar las experiencias obtenidas con el trabajo de grupos organizados a partir de 1982, para lo cual se realizó una revisión bibliográfica, con una perspectiva histórica, identificando cinco etapas por las que ha incursionado el modelo GGAVAT (Figura 1), las cuales se describen a continuación.

Figura 1: Línea del tiempo del Modelo GGAVATT

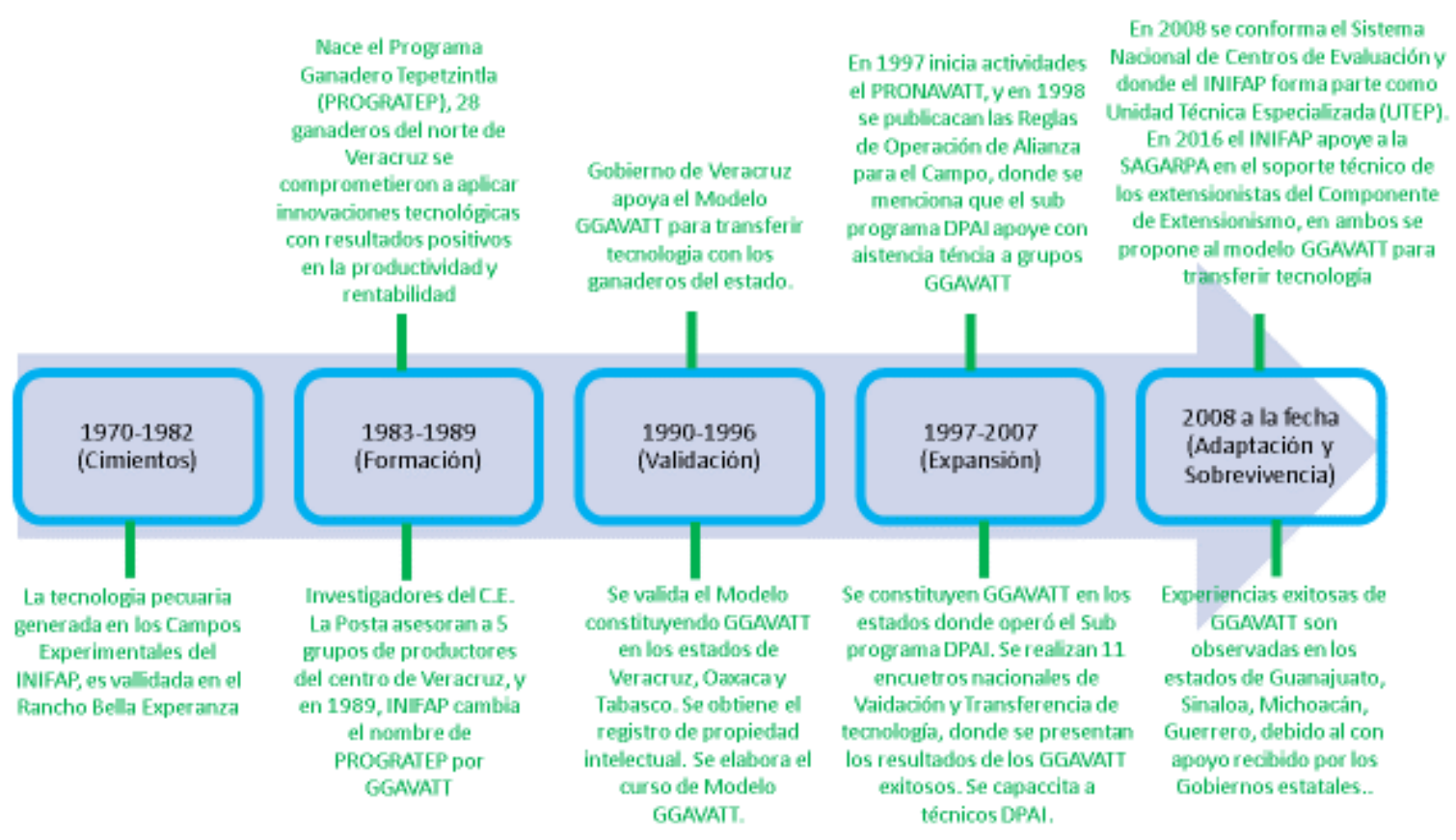




\section{Poniendo los cimientos: 1970 a 1982}

En 1970 inicia la transformación tecnológica del Rancho "Bella Esperanza" (RBE) ubicado en la Huasteca Veracruzana, con base en las recomendaciones del Campo Experimental "La posta", de Paso del Toro (CE La Posta), Veracruz ${ }^{(1,2)}$. La utilización de toros de alto valor genético mediante la Inseminación Artificial (IA) había dado buenos resultados en Estados Unidos $^{(3)}$, de igual manera, en el CE La Posta, el Holstein, Suizo Pardo y el Jersey, comprobaron su adaptación a un sistema semi estabulado(4), y con esa idea, en el RBE se cruzó ganado Brahman con Holstein, se mejoró el manejo del ganado y de los potreros a la par que la genética, y se instaló el registro sistemático de las respuestas productivas y reproductivas del hato. Un factor determinante para el éxito de este cambio tecnológico, fue el involucramiento del productor, su familia y sus trabajadores, quienes confiaron en las recomendaciones de los investigadores. Los resultados demostraron que la adopción y la aplicación de tecnología en forma ordenada permitió incrementar la producción de leche por vaca/día de $3.9 \mathrm{~kg}$ en 1971 a 6.3 en 1981, y disminuir el periodo interparto de 475 a 436 días. El cambio positivo en la producción de leche, genética del ganado, condiciones de los potreros y en general en la infraestructura, motivó para que se realizara la primera demostración técnico-práctica en el $\mathrm{RBE}^{(5)}$. Asistieron ganaderos de la Asociación Ganadera Local de Tepetzintla, otros ganaderos invitados, investigadores, profesores y autoridades del sector pecuario. Gustó lo que vieron y se comenzó a gestar la integración del grupo que a la postre se llamaría Programa Ganadero Tepetzintla (PROGATEP).

\section{Formación del modelo: 1983 a 1989}

Al conocer los avances del RBE, los ganaderos vecinos mostraron interés en utilizar la misma tecnología. Esta inquietud indujo a la integración del PROGATEP con la participación de 28 productores de la Asociación Ganadera Local de Tepetzintla, Ver. La selección de los ganaderos se hizo con base en pláticas personales, visitas a sus ranchos, interés en participar en el Programa y que la ordeña fuera una actividad importante. Los ganaderos permitieron hacer un inventario general del rancho, identificar sus animales, llevar registros de producción de leche, reproducción, ingresos y de egresos; además se comprometieron a asistir a una reunión mensual de trabajo ${ }^{(6)}$.

Se mencionó que el programa debería ser continuo, con cambios paulatinos de acuerdo con las posibilidades económicas y operativas y a las decisiones de los productores. Se definió la Asistencia técnica proporcionada por un Médico Veterinario Zootecnista del Distrito de Desarrollo Rural de Tuxpan. El INIFAP se responsabilizó de las innovaciones tecnológicas y por parte de los ganaderos se nombró a un Coordinador que fungió como enlace ante las autoridades municipales, estatales y federales, así como con la Unión Ganadera Regional. De esa forma se construyó el modelo de transferencia que propició la interacción entre 
productores, asesor técnico, investigadores e instituciones de los tres niveles de gobierno. Con la participación de los productores se elaboró el calendario de actividades con los animales y con los potreros, el programa de visitas periódicas, el calendario de reuniones mensuales (primer sábado de cada mes), el programa de pláticas técnicas para las reuniones mensuales y la evaluación anual técnica, productiva y económica (mes de abril). Se propició la participación de productores de toda la región, así como investigadores, académicos, agentes de cambio, prestadores de servicios diversos, directivos de las diversas asociaciones ganaderas y funcionarios de diferentes niveles, todos ellos relacionados con las actividades ganaderas. Se involucraron además las familias y los trabajadores de los integrantes del GGAVATT $^{(7)}$.

Además del PROGATEP, el INIFAP se responsabilizó técnicamente con otros seis grupos en el Centro del Estado de Veracruz: Programa Ganadero Jamapa, Programa Ganadero Joachín, Programa Ganadero Tres Valles, Programa Ganadero el Jilguero, Programa Ganadero La Tasajera y Programa Ganadero Porcino Jarocho. En todos ellos los resultados fueron similares al PROGATEP.

En 1989, en una reunión realizada en las oficinas del Centro de Investigación Regional del Golfo Centro, se discutió y aprobó por parte de los Investigadores del INIFAP, que el PROGATEP fuera denominado GGAVATT, con el argumento de que se trataba de un grupo ganadero que valida y transfiere tecnología. De igual forma se definió el modelo y la metodología de trabajo ${ }^{(2,8)}$. Tepetzintla pasa a la historia como el primer GGAVATT en México y se conoce como la "cuna de los GGAVATT".

\section{La validación del modelo: 1990 a 1996}

En 1990 la metodología GGAVATT se puso a disposición de todos los ganaderos del Estado de Veracruz. El PROGATEP como organización grupal, en un inicio adaptó su estructura y funciones según las políticas oficiales señaladas por el INIFAP y por el Distrito de Desarrollo Rural. Pronto la dinámica del trabajo cotidiano y de sus reuniones mensuales y anuales, fueron modificando la metodología según cambiaban las circunstancias económicas y políticas del País. La metodología del modelo GGAVATT demostró versatilidad y capacidad de adaptación ${ }^{(2)}$.

Se contaba con la documentación del grupo Tepetzintla para dar apoyo a la promoción, formación y operación de otros grupos. La promoción se realiza en las Asociaciones Ganaderas Locales, en los municipios, en los ejidos, en los Distritos de Desarrollo Rural y en general se utilizaban grupos ya formados para invitar a otros que estuvieran interesados. La formación del grupo es precedida por el diagnóstico estático aplicado directamente en los ranchos de los futuros asociados, requisito para programar la Asamblea Constitutiva, en 
donde se elige a los representantes: presidente, secretario y tesorero. En esta asamblea además se define la membresía de todos los socios. Se proponen y aceptan los componentes del grupo: ganadero, de asesoría (el profesional responsable) y el componente institucional, compuesto por las instituciones o dependencias responsables de coordinar la generación, validación y transferencia de tecnología; por último, la operación se realiza con actividades de grupo: la junta mensual, capacitación, gestoría, difusión, la evaluación anual y el encuentro nacional; así como las actividades individuales: la validación de tecnología, la aplicación en cada rancho de las actividades acordadas en el calendario de manejo para los animales y los potreros, llevar los registros productivos, reproductivos y económicos y atender los imponderables del rancho como los casos clínicos ${ }^{(6)}$.

En el seguimiento de GGAVATT en los Estados de Veracruz y Tabasco, fue importante la participación de otras instituciones de investigación y de docencia, así como las instituciones operativas relacionadas con el sector tanto del gobierno federal como del estatal. Las Uniones Ganaderas Regionales y sus respectivas Asociaciones Ganaderas Locales se incorporaron activamente. En el Estado de Veracruz, surgió el Programa Único de Grupos Ganaderos de Validación y Transferencia de Tecnología (PROGGAVATT), con el objetivo de modernizar la ganadería mediante el uso de nueva tecnología y fomentar la organización de los productores para aumentar la producción y productividad de sus ranchos sin deteriorar los recursos naturales y el ambiente agroecológico. De los 11 GGAVATT que originalmente atendía el INIFAP en Veracruz en el año de 1990, con la participación interinstitucional, este número se incrementó a 37 en 1991, a 67 en 1992 y a 79 en 1993.

En esta etapa se inician los encuentros estatales de los GGAVATT, los cuales se llevaron a cabo en el puerto de Veracruz de 1990 a 1995. Se intercambiaron experiencias y se realizaron compromisos para continuar trabajando en forma organizada. A su vez, en el estado de Tabasco se validó el modelo con dos grupos de productores en el municipio de Huimanguillo, con resultados muy similares a los obtenidos en el Estado de Veracruz.

A partir de 1990 la metodología del modelo, aplicada y supervisada hasta ese momento por el INIFAP, fue liberada para que los grupos se formaran bajo la dirección o responsabilidad técnica de quienes los productores decidieran: Uniones y Asociaciones Ganaderas, Distritos, municipios, Dirección de Ganadería, Universidades, Colegio de Médicos Veterinarios, entre otros, de esa forma, para 1995 los GGAVATT se consolidaron en los Estados de Veracruz y Tabasco $^{(7,2)}$ y se continuó con la promoción a nivel nacional ${ }^{(6)}$. 


\section{Extensión nacional del modelo GGAVATT: 1997 a 2007}

El modelo GGAVATT y sus resultados se difundieron intensamente en las Reuniones Nacionales de Investigación Pecuaria (RNIP) realizadas por el INIFAP. En 1997, además de la RNIP, se organizó en el Puerto de Veracruz, el primer Encuentro Nacional de GGAVATT, con la participación de más de mil productores de diferentes estados de la República Mexicana. Simultáneamente se organizaron Encuentros Estatales de GGAVATT y se propiciaron las giras de intercambio tecnológico en todo el País ${ }^{(1)}$. Es en este año que inicia la etapa de extensión del Modelo GGAVATT, cuando el INIFAP, para cumplir su mandato institucional de apoyar la transferencia de tecnología en materia pecuaria, conforma el Programa Nacional de Validación y Apoyo a la Transferencia de Tecnología (PRONAVATT), que fue una estrategia nacional que operó en cada estado. Se nombró un investigador del INIFAP como responsable del PRONAVATT, con el fin de promover la integración de recursos tecnológicos, intelectuales, materiales, económicos y políticos entre los sectores público, social y privado de los tres niveles de gobierno, para acelerar y masificar la transferencia de tecnología y promover el desarrollo sustentable, este programa se consideró como herramienta básica al modelo GGAVATT ${ }^{(9)}$.

También fue determinante para la consolidación del modelo, que en las reglas de operación (ROP) de la Secretaria de Agricultura, Ganadería y Desarrollo Rural publicadas en el Diario Oficial de la Federación (DOF) de 1998, en el Programa de la Alianza para el Campo (APC), se incluyera la operación del sub programa Desarrollo de Proyectos Agropecuarios Integrales (DPAI), que mencionaba que los técnicos del DPAI atendieran preferentemente a ganaderos constituidos en grupos GGAVATT ${ }^{(10)}$. El Modelo GGAVATT apareció en las Reglas de Operación de la Alianza para el Campo con algunas variaciones desde 1998 hasta 2006, como uno de los modelos organizativos promovidos por el Comité Técnico de Ganadería (COTEGAN) $)^{(11)}$, se constituyeron y operaron al menos un GGGAVATT en cada estado (excepto el Estado de México); se integraron 1,098 GGAVATT y se realizaron más de 60 Encuentros estatales y 10 nacionales; así mismo, de 2004 a 2009, mediante la Red Nacional de Validación y Transferencia de Tecnología (RENAVATT) del INIFAP, se continuó capacitando a los agentes de cambio (AC) en el modelo GGAVATT ${ }^{(9)}$.

\section{Adaptación y sobrevivencia del modelo: 2008 a la fecha}

Después del auge que representó para el modelo GGAVATT de ser incluido como uno de los modelos de transferencia a implementar en DPAI, y del mismo INIFAP con el PRONAVATT, disminuyó la expansión de GGAVATT en todo el país, en parte por el establecimiento de nuevos modelos o por la falta de recursos económicos y humano; sin embargo, en el año 2008, la SAGARPA incluye dentro del Programa Soporte la instrucción de considerar entre otros modelos al de GGAVATT, también designó al INIFAP como 
Unidad Técnica Especializada en materia pecuaria (UTEP), la cual propuso que los técnicos del Programa Soporte que atienden a productores pecuarios del país deberían de aplicar entre otros modelos al de GGAVATT, capacitarse en su metodología, además de tomar los cursos de Evaluación Diagnóstica y de Administración de Ranchos Pecuarios ${ }^{(12)}$.

En el año 2011, SAGARPA con la implementación de la Estrategia Nacional para el otorgamiento de un servicio técnico de calidad, en el marco del Componente de Asistencia Técnica para la Innovación Tecnológica, para los productores pecuarios del país que involucró a diferentes actores como los Centros Estatales de Capacitación y Seguimiento de la Calidad de los Servicios Profesionales (CECS), es decir, instituciones u organizaciones estatales, como las Universidades ${ }^{(13)}$, propició en gran medida que disminuyera el establecimiento de GGAVATT en el país, aunque hubo estados que siguieron operando con el modelo GGAVATT para su estrategia de asistencia técnica, innovación y capacitación, si bien no se ubicó un estudio que concentrara estos esfuerzos. De la revisión de las memorias de la Reunión Nacional de Investigación Pecuaria (RNIP) de $2010^{(13)}$ a la fecha, se ubicaron resultados de continuidad de GGAVATT en algunos estados, donde fue determinante el apoyo de las Fundaciones PRODUCE, como es el caso de Sinaloa y Guanajuato, y el apoyo de los gobiernos de los estados de Guerrero, Michoacán, Morelos, Nayarit, Colima, Tamaulipas, Veracruz, Campeche, Nuevo León y Chihuahua.

En el año 2013, ante la inquietud de productores organizados para la producción de carne, se estableció en el Estado de Veracruz el proyecto "Integración de la Red Bovinos Carne del Estado de Veracruz"(1). Utilizó como base la metodología GGAVATT y fue financiado del 2013 al 2014 con recursos del FIRA, la SAGARPA (Coordinación General de Ganadería) y los Productores y engordadores de bovinos del Centro de Veracruz, S.A. de C.V. (Grupo Veracarne) y del 2015 a 2016 por el Programa de Innovación, Investigación, Desarrollo Tecnológico y Educación (PIDETEC) de la SAGARPA. El proyecto finalizó en el 2017. El objetivo fue aumentar la productividad, rentabilidad y sustentabilidad de las unidades de producción de grupos de pequeños y medianos productores, a través de la asistencia técnica, la capacitación y el financiamiento; favoreciendo su organización e integración a la red de valor de carne de bovino. Participaron 20 grupos con sus respectivos asesores y se benefició a 202 ganaderos en todo el estado, con un promedio de 12 productores por grupo. El proyecto operó con la innovación del desarrollo de una plataforma informática (SIGEN-TTP Veracruz), que permitió contar con la información del diagnóstico estático al inicio de labores, después con la productiva, reproductiva, económica y de utilización de recursos crediticios. Del 2013 al 2017, las metas establecidas se cumplieron. Se constató que el modelo GGAVATT puede operar con éxito siguiendo reglas de instituciones crediticias y de organizaciones ganaderas particulares como Veracarne, además de cumplir con las reglas de operación de las dependencias oficiales. 


\section{Resultados del GGAVATT como generador de información}

Como parte de la metodología de trabajo, en los grupos se genera información desde que se aplica el diagnóstico estático (social, uso de tecnología, productivo y económico) a cada uno de los productores, igualmente se implementan registros técnicos, económicos y de usos de tecnologías, paralelo a la aplicación de los calendarios de trabajo con los animales y con los potreros; con ellos se genera información producto de la validación, utilización y adopción de tecnología. Gracias a la captura de datos directamente en los ranchos, fue posible conocer las características socioeconómicas de los productores, así como la estimación de indicadores técnicos-productivos y económicos.

\section{Características socioeconómicas de los productores}

La caracterización de los productores y de los grupos, es importante para implementar el nivel de transferencia de tecnología que se debe de aplicar y para evaluar a futuro el impacto de las acciones realizadas. En un estudio en 12 GGAVATT de la zona centro de Veracruz ${ }^{(14)}$ se encontró que la edad de los productores varía entre 20 y 80 años, en promedio 49; $94 \%$ sabe leer y escribir, $20 \%$ tiene formación profesional, $31 \%$ secundaria y preparatoria, $43 \%$ sólo tiene primaria, y $6 \%$ no posee ningún grado escolar. Las unidades de producción tienen 45.5 ha en promedio, con una carga animal de 46.6 unidades animal (UA) y sin un sistema de rotación de potreros.

Respecto a la caracterización de los grupos, en un reporte se menciona que en 1996 existían en el estado de Veracruz 79 GGAVATT. En conjunto estaban integrados por 1,288 ranchos y granjas, con 54,415 ha y alrededor de 54,000 cabezas de ganado. De los productores 473 eran pequeños propietarios y 815 ejidatarios. De los grupos, 58 eran de bovinos de doble propósito, 7 de bovinos de leche, 10 de ovinos y caprinos, 1 de porcinos, 1 apícola y 2 de aves $^{(1)}$; así mismo, para el año 2006 existían a nivel nacional 1,165 GGAVATT, que agrupaban a 17,095 productores y cerca de 1,000 agentes de cambio. Los grupos representan las principales cadenas agroalimentarias: bovinos para doble propósito, $41.1 \%$; bovinos para carne, $22.8 \%$; caprinos para carne y leche, $10.7 \%$; bovinos para leche, $10.1 \%$; avícolas, $6.4 \%$; ovinos para carne, $6.1 \%$; porcinos, $2.1 \%$; avicultura familiar, $0.5 \%$ y acuacultura, $0.2 \%{ }^{(1)}$.

El modelo GGAVATT dinamizó la adopción de tecnología e incrementó la red e interacciones sociales, proceso influenciado por las características socioeconómicas y técnico productivas $^{(15)}$. Otros autores reportan evidencias que los GGAVATT han contribuido al desarrollo de capacidades tecnológicas de los agro-empresarios cooperantes; sin embargo, el impacto en la adopción de innovaciones y rentabilidad de las UP es escaso o nulo, por lo que sugieren que se requiere mayor tiempo de intervención de los técnicos para que los beneficios sean más perceptibles ${ }^{(16)}$. 


\section{Uso de tecnología}

De acuerdo con el diagnóstico estático, los productores al inicio realizan el $30 \%$ de las tecnologías sugeridas y en el transcurso de cinco años llegan al $70 \%$. Más preciso, al año de estar organizados, 126 grupos en el Estado de Veracruz reportaron la utilización de tecnología de $48 \%$, al tercer año $63 \%$ y al quinto año $73 \%$. En otro análisis de 24 grupos se reportó $72 \pm 39 \%$ después de varios años de trabajo ${ }^{(6)}$.

Ahora bien, en un estudio que tuvo como objetivo evaluar el impacto económico y productivo de la tecnología bovina de doble propósito en Tabasco y Veracruz, se capturaron datos anuales de 1986 a 1997, en 139 ranchos incorporados a GGAVATT. Los resultados indicaron que la tecnología media superó a la tecnología baja $(P<0.05)$ en producción de leche y carne, y el ofrecer suplementos alimenticios benefició en 106 vs $44 \%$ de leche y 193 vs $81 \%$ de carne, respectivamente. El mejoramiento genético de las vacas, las praderas mejoradas y el manejo de ganado incrementaron la producción de leche en 67 y $69 \%$ y la producción de carne en 15 y $26 \%$ con la tecnología baja y media, respectivamente. Por su parte, está identificado que los productores que utilizan tecnología media, superan a los de tecnología baja $(P<0.05)$ en producción de leche, carne y beneficios sobre costos variables en 106, 44 y $81 \%$, respectivamente ${ }^{(17)}$.

En otro estudio se evaluó en Guanajuato el uso de innovaciones en grupos pertenecientes al Programa Soporte de la SAGARPA durante el periodo 2010 a 2011. Se consideró a 21 GGAVATT y 248 productores del sistema de lechería familiar ${ }^{(18)}$; se compararon tres tipos de productores: 1) 27 con nivel tecnológico bajo que aplican menos del $33 \%$ de las tecnologías, 2) 73 de nivel medio que aplican más de $33 \%$ y menos $66 \%$ y 3) 148 de nivel alto que aplican más del $66 \%$. Los resultados mostraron que el grupo uno tenía dos años de constituido y los grupos dos y tres, tres años; las prácticas que más utilizaron fueron las de salud, alimentación y manejo.

En Sinaloa se evaluó el uso de innovaciones de ocho GGAVATT pertenecientes al Programa Soporte de la SAGARPA durante el periodo 2010 a $2011^{(19)}$; se consideró a ocho GGAVATT y 121 productores, se compararon tres estratos: el primero, 38 \% con baja implementación de prácticas, que resultaron con 3.7 años de constituidos y solo usaban seis innovaciones propuestas; el segundo, $36.4 \%$ implementación media, con 4.5 años y 12.2 innovaciones y el tercero, $25.6 \%$ implementación alta, con 6.3 años y utilizaban 22.7 innovaciones.

En Michoacán se evaluó el uso de tecnología promovida por el modelo en 15 GGAVATT que participaron en el Programa de Desarrollo de Capacidades, Innovación Tecnológica y Extensionismo Rural de la SAGARPA en el ejercicio 2011-2012(20), se reporta que los productores realizaron en promedio el $77 \%$ de las actividades propuestas por el técnico y el 
$20 \%$ de los componentes tecnológicos propuestos por el INIFAP, siendo los GGAVATT de caprinos y las actividades asociadas a la prevención de enfermedades y al manejo del ganado las que presentaron los valores más altos. Se menciona que los factores determinantes en la adopción de tecnologías fueron: meses con asistencia técnica por GGAVATT, edad del productor, número de localidades, índice de marginación y número de mujeres en los grupos. Para el mismo estado, se realizó otro estudio que evaluó el impacto en la adopción de innovaciones. Se entrevistaron a 81 productores de leche de la región Ciénega de Chapala, 41 de estos productores pertenecieron a un GGAVATT durante el periodo de julio de 2008 a julio de 2011. Se evaluó el uso de 22 innovaciones: cinco de administración/organización, cuatro de reproducción y genética, cuatro de instalaciones/higiene, cuatro de nutrición y cinco de sanidad. Los resultados muestran que los ganaderos que pertenecen a un GGAVATT aplican el $51.6 \%$ de estas innovaciones y los no GGAVATT el $44.9 \%$; esta diferencia no permite concluir que exista una distancia en este sentido, entre ambos grupos de ganaderos $^{(21)}$.

En Veracruz se evaluó el uso de innovaciones de un GGAVATT constituido en 1989, y después de 10 años se entrevistó a tres estratos de ganaderos: I) los que actualmente participan en este GGAVATT; II) los que participaron; y III) los que nunca han participado. Se seleccionaron 17 variables explicativas o predictivas, de tipo social y productivas y una variable de respuesta, uso de innovaciones. Los resultados mostraron que sí existió diferencia significativa en cuanto al uso de tecnología entre los años de 1989-1990, 1990-1991 y 19931994, se reportó un incremento de uso de innovaciones, principalmente en lo que se refiere a: registros de producción y económicos, desparasitación, diagnóstico de mastitis, y de gestación y pesaje de leche; también, se notó un incremento significativo de 1990 a 1991, en lo que se refiere al pesado de leche, inseminación artificial, suplementación mineral y con subproductos, ensilaje, henificado, suplementación con forrajes de corte, fertilización de potreros y siembra de forrajes de corte. Durante 1993 y 1994, se presentó un incremento en el uso de las siguientes innovaciones: control de lactancia, suplementación con dieta balanceada y con subproductos, uso rutinario de hormonas, comercialización organizada, doble ordeño, análisis coproparasitoscópico, pastoreo intensivo y siembra de forrajes de corte $^{(22)}$.

También en Veracruz se evaluó el uso de 17 componentes tecnológicos, y la eficiencia productiva en 86 ranchos de doble propósito de 8 GGAVATT que participaron en el Programa Soporte en 2010, de los cuales el $12 \%$ tenían tres años o menos de recibir asistencia técnica, $45 \%$ tres años, $31 \%$ cuatro años y el $12 \%$ restante 5 años o más; en promedio usaban el $62 \%$ de los 17 componentes tecnológicos evaluados, sin presentarse diferencias significativas, siendo la inseminación artificial y el pesaje de destetes los componentes de menor uso y la vacunación, desparasitación, rotación de potreros, y la suplementación mineral los de mayor uso. También se reporta que si hubo diferencias significativas en cuanto 
a la producción de leche por vaca por día, donde los ranchos con más años de pertenecer a un GGAVATT son más eficientes y productivos ${ }^{(23)}$.

En el Estado de Veracruz se realizó otro estudio para conocer la dinámica de la adopción de tecnología, como una medida de evaluar el éxito del modelo GGAVATT desde una perspectiva social. Se entrevistaron a 26 productores que pertenecían a tres GGAVATT. Se observó, que la adopción de tecnología estuvo asociada al incremento del tamaño de la red e interacciones sociales, y con pocos cambios en los actores centrales. La adopción de tecnología se asocia positivamente con la educación, y es inversamente proporcional a la edad de los productores; y está influenciada por la escala de producción y manejo, los autores concluyen que el modelo GGAVATT dinamizó la adopción de tecnología, incrementando la red e interacciones sociales, proceso influenciado por las características socioeconómicas y técnico productivas de los ganaderos ${ }^{(24)}$.

En otro estudio se evaluó la eficiencia productiva y económica de los sistemas de doble propósito (SDP) y del sistema de lechería familiar (SLF) en el Estado de Veracruz, aplicando el método de investigación de un estudio de caso a cuatro GGAVATT con nivel tecnológico diferente en cada sistema de producción: para el caso del SDP un GGAVATT 1SDP de 12 integrantes, 30 años de antigüedad y aplican el $100 \%$ del paquete tecnológico; el otro GGAVATT 2SDP con 16 integrantes, 5 años de antigüedad y aplican $66 \%$ del paquete tecnológico; para el SLF, un GGAVATT 1SLF con 12 integrantes, 5 años de antigüedad y aplican el $57 \%$ del paquete tecnológico, el otro GGAVATT 2SLF con 9 integrantes, 3 años de antigüedad y aplican el $53 \%$ del paquete tecnológico. Los resultados reportados para el SDP muestran que la producción total de leche en el GGAVATT 1SDP es superior al GGAVATT 2SDP en $84 \%$; esta diferencia se origina en la adopción de una serie de prácticas tecnológicas, con diferente tiempo de respuesta, así como el tiempo aplicando el modelo, igualmente obtiene mayores ingresos, dado que sus animales tienen un precio mayor resultado de la mejora genética del hato. En el caso de los GGAVATT del SLF no se reportaron diferencias, dado que ambos GGAVATT tienen un nivel tecnológico similar ${ }^{(25)}$.

\section{Indicadores técnicos, productivos, económicos y ecológicos}

En todos los GGAVATT del país, comparando con los indicadores tradicionales, se reportan incrementos en la producción de leche del orden del 100 a $200 \%$ o más, en la producción de carne la proporción es menor, del 50 a $100 \%$, de igual manera sucede con los indicadores reproductivos y económicos.

Asociado al uso de un $70 \%$ de tecnología, en el estado de Veracruz se reportaron $286 \pm 44$ días para días de ordeño; $2,159 \pm 921 \mathrm{~kg}$ de leche por lactancia; $7.25 \pm 2.3 \mathrm{~kg}$ de leche por vaca día; $4.7 \pm 2.1 \mathrm{~kg}$ de leche por día interparto y $900 \pm 396 \mathrm{~kg}$ de leche por hectárea ${ }^{(6)}$. 
El caso Tepetzintla ejemplifica las posibilidades de incremento. Los ranchos lograron en un inicio $74 \%$ de uso de tecnología y rebasaron los 1,000 kg por lactancia; se intensificó el uso de tecnología al $95 \%$ y se rebasaron los 2,000 kg de leche por lactancia. El liderazgo de los productores, técnicos e instituciones permitió la adopción de alta, difícil y costosa tecnología; posteriormente, aunque descendió el uso de tecnología al 74 \%, se logró rebasar los 3,000 kg por lactancia; por último, con $85 \%$ de tecnología y con la utilización de altos niveles de alimento concentrado, se lograron 4,000 kg por lactancia, y al disminuir el alimento, se estabilizaron en $3,500 \mathrm{~kg}^{(6)}$.

En el año 2006 se evalúo en Sinaloa el impacto económico que generó el uso de componentes tecnológicos en el área de la alimentación animal en el proyecto GGAVATT Sinaloa, con la tecnología generada para los GGAVATT los rendimientos de forraje seco en el cultivo de sorgo, fue de 7.0 contra 3.9 t de MS/ha, que representó un incremento del $79 \%$, el efecto en la producción fue el incremento de la producción de leche por vaca por día en $60 \%$, y el período de lactancia de 180 días con el sistema tradicional a 210 con la tecnología INIFAP ${ }^{(26)}$. En Veracruz se analizaron los promedios de 24 GGAVATT de bovinos de doble propósito y se encontraron rentabilidades sobre costos variables (RSCV) del orden de $85 \pm 49 \%$ y para la rentabilidad sobre inversión (RSI) solo el $3.5 \pm 3.6 \%{ }^{(6)}$.

Desde sus inicios, uno de los propósitos que se promueven en los grupos, es el cuidado y mejora de los recursos naturales. En este sentido, en un estudio que se realizó en los años 2005 y 2006 en la zona centro del estado de Veracruz en cuatro GGAVATT con sistema de lechería familiar (SLF) de la zona montañosa y en ocho con sistemas de bovinos de doble propósito (SBDP), se obtuvo información mediante entrevistas y recorridos de campo para realizar muestreos de vegetación en cercos vivos, fragmentos de vegetación. Se inventariaron un total de 66 especies de árboles y arbustos en uso: en los cercos vivos se reportan 37 especies, en las orillas de los ríos y arroyos (vegetación riparia) 33 especies y se identificaron a su vez 37 especies de pastos. Los ganaderos también reportaron la introducción de especies

y cultivos exóticos, así como frutales ${ }^{(15)}$, lo anterior muestra el cuidado de la vegetación que los ganaderos incorporados a los GGAVATT realizan.

\section{Ejemplos de éxito del modelo GGAVATT}

En el 2005 el INIFAP realizó un estudio para identificar en ese entonces, las contribuciones del modelo GGAVATT al desarrollo de la ganadería ${ }^{(27)}$, se visitó a 16 estados: Veracruz, Guanajuato, Campeche, Coahuila, Tabasco, Guerrero, Sinaloa, Puebla, Yucatán, San Luis Potosí, Durango, Morelos, Sonora, Nayarit, Baja California Sur, Nuevo León. Se entrevistaron 33 productores de 10 estados, 19 asesores de GGAVATT y a tres investigadores que generaron del modelo. El $94.0 \%$ de los productores coincidió que el factor más importante de éxito fue trabajar como grupo organizado, el $57.6 \%$ dijo que la transferencia 
de tecnología, el $54.5 \%$ mencionó los apoyos que se lograron como grupo organizado y el $45.0 \%$ se refirió al liderazgo profesional e institucional; solo el $27.3 \%$ mencionó como factor importante el incremento en la producción de sus ranchos.

El $37 \%$ de los asesores opinó que el incremento en la producción es uno de los principales factores, el $32 \%$ coincidió en la unidad y unión entre los productores, el $26 \%$ en la organización del grupo y el $21 \%$ los registros productivos y económicos, las ganas de trabajar y el interés por la nueva tecnología; el $16 \%$ mencionó la aplicación de la metodología, las buenas relaciones y un ambiente de competencia sana; y solo el $10 \%$ hizo alusión a el uso de un calendario de actividades, la capacitación, la asesoría en los ranchos, el compañerismo, el convencimiento de las bondades de la adopción de tecnología, el diagnóstico estático, el convencimiento y el aprendizaje.

Los investigadores coincidieron en que es un modelo totalmente replicable y que propicia el cambio de todos los participantes; en menor proporción mencionaron que se propicia el liderazgo institucional, la organización, el trabajo en grupo, la convivencia social, la capacitación, la obtención de información técnico productiva y económica, la disposición a la nueva tecnología, la apropiación del modelo por parte de los productores, el interés, la motivación, la amistad y en general el humanismo.

Los factores en que coincidieron los productores, asesores e investigadores fueron el trabajo como grupo organizado, la capacitación, la información productiva, la motivación y el interés por la nueva tecnología.

En el caso Tepetzintla, la adopción de nueva tecnología incrementó la producción, los ingresos y mejoró el capital tangible (praderas, ganado, construcciones y equipo), con ello se cumplió parte del objetivo inicial ${ }^{(6)}$; sin embargo, a lo largo de 25 años de trabajo, emergieron capitales intangibles que favorecieron un cambio importante en todos los participantes: en principio se respetó el "capital tácito cognitivo" propio de la huasteca, región con amplia cultura agrícola y ganadera heredada de sus ancestros, así surgieron verdaderos líderes motivadores del cambio tecnológico: productores, asesores e investigadores; se mantuvo intacto su "capital cultural": tradiciones, mitos, creencias, lenguaje (nahuatl), manifestaciones de arte, todo en conjunto favoreció la actitud hacia el trabajo; se desarrolló el "capital institucional": además del INIFAP, se involucraron los gobiernos estatal y federal, otras organizaciones ganaderas, instituciones de investigación y de docencia, de crédito, capacitación, desarrollo y de fomento. La metodología fue incluyente desde un principio. En opinión de los productores, se incrementó el "capital psicosocial"; gracias a la organización el grupo obtuvo reconocimiento y respeto con base en valores, amistad verdadera, compromiso de trabajo, competencia leal, participación individual y familiar (destaca el involucramiento en el trabajo de las mujeres) y habilidad para precisar y solucionar problemas; se propició el desarrollo del "capital social", el GGAVATT empezó como una 
figura asociativa "a la palabra", un grupo de amigos y según las circunstancias y la versatilidad del trabajo, se integraron figuras asociativas legales y familiares; sin duda el desarrollo del "capital humano" es el más importante, se incrementó la capacidad de autogestión, la seguridad para comunicarse técnicamente, se formó una generación de hijos universitarios que se involucraron con el grupo y en el trabajo de la región (siete mujeres y siete hombres, entre ellos seis médicos veterinarios zootecnistas), por último, los productores fueron reconocidos nacionalmente al ser considerados como "la cuna de los GGAVATT".

Guanajuato es un estado ejemplo de constancia con la aplicación del modelo GGAVATT. Desde su primer grupo de lechería familiar "La Labor" establecido en el municipio de Apaseo el Grande ${ }^{(28)}$, han mantenido un promedio de 77 grupos por año: $26.0 \%$ de bovinos productores de leche, $20.3 \%$ de caprinos, $19.1 \%$ bovinos productores de carne, $16.3 \%$ ovinos, $10 \%$ porcinos y $8.3 \%$ de abejas y se han beneficiado anualmente un promedio de 1,352 ganaderos. De 1999 a la fecha, los grupos contaron con el apoyo de diferentes Programas: en el año 2002 con el programa DEPAI establecieron 74 GGAVATT, el aporte principal fue el pago de asesores que continúo hasta el año 2006; del 2007 al 2012 participaron con el Programa Soporte y del 2012 al 2018 con el programa de Extensionismo. En el año 2019, la Federación retira sus recursos de apoyo y entonces el Estado de Guanajuato paga al $100 \%$ el salario de los asesores; además, de 1998 a 2007, contaron con el apoyo de la Fundación Guanajuato Produce (FGP). Además del beneficio de los programas, los grupos se favorecieron con la participación de los investigadores del INIFAP, la Universidad de Guanajuato, el Instituto Tecnológico de Roque, laboratorios farmacéuticos y otras empresas privadas.

La percepción de mejora que los productores han observado al estar en GGAVATT, han roto una inercia muy arraigada en los productores del campo en México, que es asociar un programa gubernamental con la obtención de apoyos, de tal forma que los productores han privilegiado la asistencia técnica y capacitación aún sobre los apoyos en equipamiento, infraestructura y semovientes, como lo evidencia un análisis de su cambio de actitud ${ }^{(29)}$.

Del año 2002 al año 2020 se han realizado 14 encuentros estatales de transferencia de tecnología, con una asistencia por evento de más de 1,000 productores. De la información presentada en estos eventos, destaca la del GGAVATT "Caprinocultores Unidos de Guanajuato", la producción por lactancia supera a los 1,100 kg. Por el valor genético de sus animales, estos productores han sido proveedores de sementales en los programas de apoyo gubernamentales, beneficiando a cientos de caprinocultores del estado y seguramente han contribuido para que Guanajuato aumente su producción de leche de cabra, de 2010 a 2019 en un $68.9 \%$, pasando de 24,980 a 42,196 t, con ello Guanajuato escaló del 3er al 2do lugar nacional en producción de leche de cabra ${ }^{(30)}$. 
Sinaloa es otro estado donde se aplicó el modelo GGAVATT por un periodo de tiempo considerable, alcanzado impactos productivos, tecnológicos, económicos y sociales ${ }^{(31)}$. Se formaron 499 GGAVATT, que agruparon a 4,661 productores a su vez atendidos por 185 técnicos ${ }^{(31)}$. La cadena prioritaria fueron los bovinos de doble propósito. Participaron en varios programas: DEPAI, SINDER, PEAT, UTEP, SOPORTE, además de los propios del gobierno del estado. Este trabajo continuó hasta el periodo 2013-2014 ${ }^{(32)}$. A partir del año 2014, ya sin el apoyo federal, la Fundación Produce de Sinaloa se responsabilizó del trabajo y continuidad de los grupos.

A lo largo de los encuentros nacional del PRONAVATT, los GGAVATT del Estado de Sinaloa estuvieron presentes como "casos exitosos", reportados en sus respectivas memorias, en el año 2000, fueron los GAVATT "Zavala 1" y "El Sacrificio" y en la del 2003 el GGAVATT "Chinobampo", todos del sistema de bovinos de doble propósito ${ }^{(31)}$.

Veracruz como estado, al igual que los estados anteriores, ha trabajado intensamente con el modelo GGAVATT, de 1982 a 2006 se reportaron 409 grupos, de ellos el $38 \%$ permaneció integrado solo un año, un $17 \%$ de cuatro a cinco años y un $14 \%$ más de cinco. La meta es que los grupos permanezcan integrados por lo menos de cuatro a cinco años, el caso Tepetzintla es ejemplar, pues cuenta con más de 35 años de constituido. Otros GGAVATT superaron la barrera de los 10 años e incluso la de 15, por lo general fueron los asociados con el INIFAP como institución responsable ${ }^{(6)}$.

En el estado de Tamaulipas se constituyeron 34 GGAVATT en el año de 2009, cada uno en promedio con 20 productores y atendidos por su respectivo prestador de servicios profesionales (PSP). Los grupos se ubicaron en 16 de los 43 municipios del estado (37\%), destaca Mante con siete grupos y Aldama con cuatro, el resto con uno o dos. En el 2010 y 2011, la UTEP apoyó con la capacitación, supervisión y evaluación de los PSP. Participaron 559 productores de las diferentes especies-producto existentes. Se levantaron encuestas específicas por productor en: bovinos carne (16); doble propósito (7); caprinos (5); apícolas (4) y ovinos (2); de esa forma fue posible registrar 66 tecnologías: para el área de Alimentación y Forrajes se incorporaron 18, $23.7 \%$; en Genética cuatro, $6.1 \%$; en Reproducción Animal siete, 10.6 \%; en Sanidad 11, $16.7 \%$; en Administración tres, $4.5 \%$; en otros complementos (uso de implantes y aditivos) tres, $4.5 \%$ y en la industrialización de la miel fueron dos incorporaciones tecnológicas, $3.0 \%$. Parece fácil, pero trabajar con productores de cinco especie-producto diferentes, constituyó un buen reto ${ }^{(32)}$.

\section{Impactos ambientales, económicos, productivos y sociales del modelo GGAVATT}

En el aspecto ambiental se logró incrementar el establecimiento de cercos vivos en los GGAVATT de lechería familiar y de doble propósito del estado de Veracruz ${ }^{(15)}$, con un 
impacto positivo en la vegetación y el bienestar animal al tener más espacios de sombra en los potreros. En los GGAVATT de Sinaloa se promovió el uso de componentes tecnológicos en el área de alimentación animal: elaboración y uso de bloques multinutricionales, establecimiento y manejo de praderas, uso de cercos vivos, nuevas opciones de especies forrajeras y ensilajes, disminuyendo la pérdida de suelo en un $88 \%$ y de agua en un $50 \%$ (26,31).

Una de las tecnologías que se promueven con el modelo GGAVATT es el uso de registros técnicos y económicos, del análisis de la información generada con estos registros se ha logrado evaluar el impacto productivo y económicos de la adopción de tecnología en las unidades de producción que participan en un GGAVATT. En un estudio donde se analizó la información de 206 ranchos de doble propósito en los estados de Campeche, Colima, Nayarit, Sinaloa y Veracruz, que recibieron asistencia técnica y capacitación pecuaria durante los ejercicios fiscales 2011 y 2012, se identificaron dos tipos de productores: uno con nivel tecnológico bajo, $76 \%$, que aplican en promedio el $33 \%$ de innovaciones propuestas y productores con nivel tecnológico intermedio, $24 \%$, que usan en promedio el $66 \%$ de innovaciones. Al comparar las variables de productividad y rentabilidad de los dos tipos de productores, se encontró que el tipo de productores con más uso de innovaciones obtienen 1.86 litros de leche más por vaca por día y $8 \%$ más de rentabilidad ${ }^{(33)}$.

Sin duda, uno de los mayores impactos del modelo GGAVATT ha sido la adopción de innovaciones y con ello un impacto social al desarrollar capacidades tecnológicas y de organización de los productores, como lo muestran los estudios realizados con productores de leche de Michoacán ${ }^{(21)}$, y de Guanajuato ${ }^{(18)}$ con productores de bovinos de doble propósito de Veracruz ${ }^{(23,24,25)}$, y de Sinaloa ${ }^{(19)}$, en donde se menciona que las unidades de producción que han participado en el modelo GGAVATT han incrementado el uso de innovaciones.

\section{Perspectivas del modelo GGAVATT}

La adaptación y permanencia del GGAVATT como un modelo para la organización de los productores desde hace más de 30 años, hace suponer que, si se crean nuevamente las condiciones y apoyos apropiados, es posible que resurja la dinámica de trabajo de los grupos, con los productores, los profesionales, las dependencias y las instituciones. Este supuesto se fundamenta en lo siguiente: el modelo GGAVATT se ha adaptado a la gran diversidad de programas y proyectos en los tres niveles de gobierno ${ }^{(1)}$, por su parte, el INIFAP ha tenido protagonismo en la mayoría de ellos. En la cronología destacan en 1986 los Grupos de Intercambio Técnico de los Distritos de Desarrollo Rural (DDR), el INIFAP se responsabilizaba de los paquetes tecnológicos; de 1990 a 1995 se establece el Programa de Proyectos de Investigación y Extensión agropecuaria y Forestal (PIEX), con la participación de los DDR, FIRA, y BANRURAL, colateral con FIRCO; en 1995 se integra la Coordinación 
Nacional de Fundaciones produce (COFUPRO) con sus respectivas Fundaciones Produce en los estados; en1996 la SAGARPA queda como normativa y los estados como operativos, se forman las Comisiones de Desarrollo, con la participación de INCA Rural, INIFAP, FIRCO y la agencia de Comercialización y Desarrollo (ASERCA); de igual forma, da inicio el Programa Alianza para el campo con el Sistema Nacional de Capacitación y Extensión (SINDER) y el Programa Elemental para la Asistencia Técnica (PEAT), con buena participación del INCA Rural, además se estableció el Programa de Desarrollo Rural sustentable (DRS); en 1998 y muy importante para los GGAVATT, se estableció el Programa de Desarrollo de Proyectos Agropecuarios Integrales (DPAI) buscando el desarrollo de cuencas productivas; en 2001 se fusionan SINDER y PEAT en el Programa de Extensión y Servicios Profesionales (PESPRO), que a su vez se transforma en el Programa de Desarrollo de Capacidades (PRODESCA); en 2003 todos los programas se fusionan y se crea el Fomento Ganadero y Desarrollo de Proyectos Integrales (DPAI) en el cual continua el GGAVATT, colateral se establece con cobertura nacional el Programa de Estímulos a la Productividad Ganadera (PROGAN) y finalmente, en 2008, la Coordinación General de Ganadería invita al INIFAP a participar en el Sistema Nacional de Centros de Evaluación, como Unidad Técnica Especializada (UTEP), mediante a la cual se siguió apoyando a grupos de ganaderos con cerca de 1,000 PSP. Como se mencionó, en todos los programas y proyectos, el GGAVATT encontró la posibilidad de participar y eso lo hace en una perspectiva positiva, un candidato a seguir participando.

\section{Conclusiones}

El modelo GGAVATT es una tecnología de proceso que brindó a los investigadores del INIFAP, la oportunidad de participar en los ranchos de productores organizados, para validar, transferir e innovar tecnologías generadas en los campos experimentales. El GGAVATT es un modelo participativo, que se origina de la participación de los productores con los investigadores. Aumenta la productividad y rentabilidad de las UPP. Genera capital tangible e intangible y aumenta el capital social, intrínseco, horizontal y vertical de los productores.

El GGAVATT se puede utilizar con éxito en cualquier sistema de producción pecuaria y región agroecológica del país, y es posible que sea un buen conducto para mejorar los recursos naturales y reducir el impacto del cambio climático. El modelo cuenta con una metodología de trabajo debidamente documentada, que la hace creíble y favorece la voluntad de replicarla en todos los estados de la República, por lo tanto, existe la posibilidad de que sea el eje de la transformación tecnológica y desarrollo integral de la ganadería nacional. 


\section{Agradecimientos}

Los autores de este escrito agradecen a todos los técnicos y productores que participaron en desarrollar la tecnología de proceso del Modelo GGAVATT y en particular al MVZ Jesús Manuel Pérez Saldaña ${ }^{+}$y al productor Caín Román Ponce ${ }^{+}$por su invaluable compromiso con la validación, innovación y transferencia de tecnología.

\section{Conflictos de interés}

Los autores declaran que no tienen conflictos de interés con respecto al trabajo presentado en este reporte.

\section{Literatura citada:}

1. Román PH, Rodríguez ChMA, Aguilera SR, Rivera VGH. La transferencia de tecnología en las regiones tropicales de México. En: Rodríguez RO editor. Estado del arte sobre investigación e innovación tecnológica en ganadería bovina tropical. Segunda ed. Ciudad de México, México: RED GATRO CONACYT; 2018:331-343.

2. Román PH, Bueno DH, Aguilar BU, Pérez SJM, Rodríguez ChMA, Koppel RE. Manual del Modelo GGAVATT. Folleto técnico Núm. 27. INIFAP Produce. Veracruz. México; 2001:39-46.

3. Carter JA, Bellow S, Meintjes M, Pérez O, Ferguson E, Godke RA. Transvaginal ultrasound-guided oocyte transpiration for production of embryos in vitro. Arch Tierz Dummerstorf 2002;45(1):99-108.

4. Román PH, Cabello FE, Wilcox ChJ. Producción de leche de vacas Holstein, Suizo Pardo y Jersey en clima tropical. Téc Pecu Méx 1978;34:21-33.

5. Rodríguez ChMA, Román PH, Troncoso AH, Vázquez PC, Saldaña AR. Evaluación del programa ganadero Tepetzintla como un modelo de validación y transferencia de tecnología pecuaria para ganado bovinos de doble propósito en la Huasteca veracruzana. Vet Mex 1991;22(2): 230.

6. Rodríguez ChMA. Factores tangibles e intangibles que contribuyen a la evolución, permanencia e impacto del modelo GGAVATT en el estado de Veracruz, México (19822007) [tesis doctoral]. Veracruz, México. Colegio de Posgraduados-Campus Veracruz; 2010. 
7. Rodríguez ChMA. Evaluación del programa ganadero Tepetzintla como un modelo de validación y transferencia de tecnología pecuaria para ganado bovino del trópico en la Huasteca Veracruzana. [Tesis de maestría]. México, DF: Universidad Nacional Autónoma de México; 1990.

8. Rodríguez ChMA, Román PH, Pérez SJ, Bueno DHM, Aguilar BU. El modelo GGAVATT, estrategia de validación y transferencia de tecnología pecuaria. En: Octava Reunión Científico-Tecnológica Forestal y Agropecuaria. Veracruz, Ver. Mex.1995:226-232.

9. Vázquez GR, Rivera MJA, González SA, Cabrera TEJ. La transferencia de tecnología bovina en las regiones tropicales de México. En: Rodríguez RO editor. Estado del arte sobre investigación e innovación tecnológica en ganadería bovina tropical. Primera ed. Ciudad de México, México: RED GATRO CONACYT; 2015:251-272.

10. SAGARPA. Proyecto de Evaluación Alianza para el Campo 2006. Análisis de Políticas. Alianza para el Campo: Hacía una nueva etapa. Propuesta para el período 2007-2012. 2007:75. http://www.fao.org/3/a-bc941s.pdf. Consultado 26 Sep, 2020.

11. Diario Oficial de la Federación. Reglas de operación de la SAGARPA, 1998-2012. www.https://dof.gob.mx/. Consultado 18 Sep, 2020.

12. Flores MAB, Vázquez GR. Desempeño y asimilación de la estrategia UTEP en el período 2010 [resumen]. XLVII Reunión Nacional de Investigación Pecuaria. León, Guanajuato 2011:218.

13. Flores MAB, Vázquez GR. Contraste del desempeño en la implementación de la estrategia de la UTEP en los años 2010, a cargo de INIFAP y 2012 a cargo de los CECS [resumen]. Reunión Nacional de Investigación Pecuaria. Veracruz. 2013:202.

14. Contreras HA, Osorio RML, Aguilar BU, Román PH, Espinosa GA, Martínez RJL, Trujillo JE. Evaluación del impacto de la ganadería tropical en el centro de Veracruz. En: La encrucijada de México rural. Contrastes desiguales en un mundo desigual. Coordinadores: Contreras HA, Kauffer MEF. Tomo VI. Recursos naturales, institucionales locales y políticas ambientales: las encrucijadas de la conservación. AMER. Primera ed. México 2011.

15. Pulido-Albores AR. An evaluation of the impact of a technology transfer programme on dual purpose cattle production systems in Veracruz, Mexico [doctoral thesis]. England: University of London; 2001.

16. González-Estrada, A. Industrialización y transnacionalización de la agricultura mexicana. Rev Mex Cienc Agr 2016;7(3):693-707. 
17. Espinosa GJA, Matus GJA, Martínez DMA, Santiago CMJ, Román PH, Bucio AL. Análisis económico de la tecnología bovina de doble propósito en la Región del Golfo. Agrociencia 2000;34(5):651-661.

18. Vélez IA, Espinosa GJA, Omaña SJM, González OTA, Quiroz VJ. Adopción de tecnología en unidades de producción de lechería familiar en Guanajuato, México. Rev Actas Iberoamer Conserv Anim 2013;(3):89-96.

19. Cuevas RV, Cervantes EF, Espinosa GJA, Aguilar ÁJ, Loaiza MA. Factores que determinan el uso de innovaciones tecnológicas en la ganadería de doble propósito en Sinaloa, México. Rev Mex Cienc Pecu 2013;4(1):31-46.

20. Zalapa RA, Carrera VJA, Morales VJG, Arreola ZJM. Análisis del programa de asistencia técnica pecuaria en el estado de Michoacán. Sitio Argentino de Producción Animal. Documento 156, 2013. http://www.produccionanimal.com.ar/informacion_tecnica/origenes_evolucion_y_esta disticas_de_la_ganaderia/156-Zapala. Consultado 10 Oct, 2020.

21. Ponce MF, Álvarez BD, Ceja TLF. Modelo GGAVATT y redes de innovación en la cuenca lechera Ciénega de Chapala, Michoacán. Rev Mex Cienc Agr 2016;7(3):545558.

22. Galindo GG. Uso de innovaciones en el grupo de ganaderos para la validación y transferencia de tecnología “Joachin”, Veracruz, México. Terra 2001;9(4):385-392.

23. Valdovinos TME, Espinosa GJA, Vélez IA. Innovación y eficiencia de unidades bovinas de doble propósito en Veracruz. Rev Mex Agroneg 2015;XIX(36):1306-1314.

24. Cárdenas BE, Gallardo LF, Nuñez EJF, Asiaín HA, Rodríguez ChMA, Velázquez BG. Redes de innovación en los grupos ganaderos de validación y transferencia de tecnología en México. ASyD 2016;13:237-255.

25. Vázquez SE, Aguilar BU, Villagómez CJA. Comparación de la eficiencia productiva y económica de grupos ganaderos organizados de doble propósito y de lechería familiar/semiespecializada. Cienc Admin 2016;1(1):226-237.

26. González EA, Wood S. Impactos económicos de tecnologías para el campo mexicano. SAGARPA INIFAP. Libro científico. Núm 1. Chapingo, México 2006:341-355.

27. INIFAP. Contribuciones del modelo GGAVATT al desarrollo de la ganadería Testimonios. Publicación Especial No. 1. CENID-Microbiología. México. 2005. 
28. González, OTA, Peña, LV, Espinosa GJA. GGAVATT de lechería familiar, la labor. Primera evaluación. Instituto Nacional de Investigaciones Forestales, Agrícolas y Pecuarias. Campo Experimental Bajío, Celaya, Gto., México. Publicación Especial. 2001;(1):1-27.

29. Bustos CDE, Espinosa GJA, González OTA, Tapia NCA. GGAVATT en el estado de Guanajuato. Análisis del cambio de actitud en los productores. Publicación Técnica No.1. SAGARPA. INIFAP. CIRCE. Querétaro, México 2008;5-61.

30. Agenda de innovación de Guanajuato, Conacyt. 2014.

31. Loaiza MA. Transferencia de tecnología a grupos de ganaderos en Sinaloa. INIFAP. Primera ed. Fundación Produce Sinaloa, A.C. Colección: Resultados de proyectos. Culiacán, Sinaloa, México. 2014.

32. Guarneros AR, Suárez MEJ, Rosales AJ. Tecnologías incorporadas en los programas de asistencia técnica y capacitación en Tamaulipas. Libro de memorias, Extensos $23^{\circ}$ Encuentro Nacional de Investigación Científica y Tecnológica del Golfo de México. 2012.

33. Espinosa GJA, Vélez IA, Góngora GSF, Cuevas RV, Vázquez GR, Rivera MJA. Evaluación del impacto en la productividad y rentabilidad de la tecnología transferida al sistema de bovinos de doble propósito del trópico mexicano. Rev Trop Subtrop Agroecosystems 2018;21:261-272. 